\title{
Chern-Simons Theories on Noncommutative Plane
}

\author{
Dongsu Bak, ${ }^{a}$ Kimyeong Lee, ${ }^{b}$ and Jeong-Hyuck Park ${ }^{b}$ \\ Physics Department, University of Seoul, Seoul 130-743 Korea ${ }^{a}$ \\ Korea Institute for Advanced Study, 207-43, Cheongryangri-dong, Dongdaemun-gu, Seoul 130-012, Korea ${ }^{b}$
}

(November 1, 2018)

\begin{abstract}
We investigate $U(N)$ Chern-Simons theories on noncommutative plane. We show that for the theories to be consistent quantum mechanically, the coefficient of the Chern-Simons term should be quantized $\kappa=n / 2 \pi$ with an integer $n$. This is a surprise for the $U(1)$ gauge theory. When uniform background charge density $\rho_{e}$ is present, the quantization rule changes to $\kappa+\rho_{e} \theta=n / 2 \pi$ with noncommutative parameter $\theta$. With the exact expression for the angular momentum, we argue in the $U(1)$ theory that charged particles in the symmetric phase carry fractional spin $1 / 2 n$ and vortices in the broken phase carry half-integer or integer spin $-n / 2$.
\end{abstract}

PACS numbers : 11.15.Tk,11.30.-j,11.10.Kk

Recently there has been a considerable interest in the noncommutative gauge theories with Yang-Mills kinetic term [1]. The Wilson loop line segments and the covariant position operators play a crucial role in defining the covariant semi-local densities [2 2 . The energy momentum tensors are gauge covariant and so the issue of the gauge invariant local operator has been discussed in many directions.

The Chern-Simons gauge theories on noncommutative plane have been studied also recently [5 7 . There was a claim that there is no need for the Chern-Simons coefficient in the noncommutative case [8].

In this letter, we show that the Chern-Simons coefficient should be quantized if the noncommutative $U(N)$ Chern-Simons theories are consistent quantum mechanically. This is true even for the $U(1)$ theory. One can also introduce an additional uniform charge as the background, in which case only a linear combination of the background charge and the Chern-Simons coefficient is quantized. We also find the conserved angular momentum and calculate it for any rotationally symmetric configuration. The result is identical to the commutative case, implying that the spin-statistics theorem holds even for the noncommutative field theories.

Recently Susskind [9] argued that the noncommutative version of the $U(1)$ Chern-Simons theory at level $n$ is exactly equivalent to the Laughlin theory of the filling fraction $1 / n$. In this argument, the quantization of the Chern-Simons coefficient is essential. The present paper provides the proof of the quantization.

The noncommutative plane is defined by the coordinates $(x, y)$ satisfying the commutative relation

$$
[x, y]=i \theta .
$$

We choose $\theta>0$ without loss of generality. The Hilbert space of harmonic oscillator is defined by the annihilation and creation operators

$$
a=\frac{x+i y}{\sqrt{2 \theta}}, \quad \bar{a}=\frac{x-i y}{\sqrt{2 \theta}}
$$

which satisfy $\left[a, a^{\dagger}\right]=1$. The space integration $\int d^{2} x$ becomes $2 \pi \theta$ Tr.

For the $U(N)$ gauge group, the gauge field $A(x)$ is hermitian matrix valued operator on the noncommutative space. For simplicity, we just include a charged matter field $\phi(x)$. The action for the theory is the sum of the Yang-Mills action, the Chern-Simons action, and the matter action. The Yang-Mills action is

$$
S_{Y M}=\int d t 2 \pi \theta \operatorname{Tr}\left(-\frac{1}{4 e^{2}} \operatorname{tr} F_{\mu \nu} F^{\mu \nu}\right),
$$

where $F_{\mu \nu}=\partial_{\mu} A_{\nu}-\partial_{\nu} A_{\mu}-i\left[A_{\mu}, A_{\nu}\right]$. The small tr is over the $N$ by $N$ group indices. The Chern-Simons action [10] is

$$
S_{C S}=\int d t 2 \pi \theta \operatorname{Tr} \frac{\kappa}{2} \epsilon^{\mu \nu \rho} \operatorname{tr}\left(A_{\mu} \partial_{\nu} A_{\rho}-\frac{2 i}{3} A_{\mu} A_{\nu} A_{\rho}\right) .
$$

The matter action is

$$
S_{\text {matter }}=\int d t 2 \pi \theta \operatorname{Tr} \operatorname{tr}\left(-\nabla_{\mu} \phi \nabla^{\mu} \bar{\phi}-U(\phi, \bar{\phi})\right),
$$

where the covariant derivative is $\nabla_{\mu} \phi=\left(\partial_{\mu}-i A_{\mu}\right) \phi$.

Under the local $U(N)$ gauge transformation $g=e^{i \Lambda(x)}$, the gauge field $A_{\mu}$ transforms as $A_{\mu}(x) \rightarrow g A_{\mu} \bar{g}-i \partial_{\mu} g \bar{g}$, and $\phi \rightarrow g \phi$. The Yang-Mills and matter actions are manifestly invariant under this transformation. However, the Chern-Simons action is not manifestly invariant. Rather its change is

$$
\Delta S_{C S}=i \pi \kappa \theta \int d t \operatorname{Tr} \operatorname{tr} \epsilon^{\mu \nu \rho} \partial_{\mu}\left(\partial_{\nu} g A_{\rho} \bar{g}\right)-4 \pi^{2} \kappa \mathcal{N},
$$

where

$$
\mathcal{N}=\frac{1}{24 \pi^{2}} \int d t 2 \pi \theta \operatorname{Tr} \operatorname{tr} \epsilon^{\mu \nu \rho}\left(\bar{g} \partial_{\mu} g \bar{g} \partial_{\nu} g \bar{g} \partial_{\rho} g\right) .
$$

This quantity $\mathcal{N}$ is the analogue of the 'topological number' for the mapping from $S^{3} \rightarrow S U(2)$. As $\partial_{i} g=\left[\hat{\partial}_{i}, g\right]$ with $\hat{\partial}_{i}=\frac{i}{\theta} \epsilon_{i j} x^{j}$, the topological number becomes 


$$
\mathcal{N}(g)=-\frac{i}{4 \pi} \int d t \operatorname{Tr} \operatorname{tr}\left(\bar{g} \partial_{t} g[\bar{g}[a, g], \bar{g}[\bar{a}, g]]\right) .
$$

As we make successive gauge transformations which go to the identity quickly at infinity, their topological numbers add up. Thus, we see that $\mathcal{N}\left(g_{1} g_{2}\right)=\mathcal{N}\left(g_{1}\right)+\mathcal{N}\left(g_{2}\right)$.

The gauge transformation is $g(x)=e^{i \Lambda(x)}$ with a hermitian matrix valued function $\Lambda(x)$. As $\Lambda$ is hermitian also as an operator on the Hilbert space, we can find a unitary transformation $U(x)$ which diagonalize $\Lambda$ and so $g$. That is $g=U e^{i \Lambda_{D}} \bar{U}$ with a diagonal $\Lambda_{D}$. The topological charge would not change with this diagonalization. Thus we need to calculate the topological number for $g=e^{i \Lambda_{D}}$. The general expression for the diagonal function $\Lambda_{D}(x)$ is

$$
\Lambda_{D}(x)=\sum_{l} \operatorname{diag}\left(f_{1 l}(t), f_{2 l}(t), \ldots, f_{N l}(t)\right)|l\rangle\langle l|,
$$

where $f_{a l}(t)$ are the real functions of time only. The contribution of each diagonal element is independent and adds to the topological charge,

$$
\mathcal{N}(g)=\frac{1}{2 \pi} \sum_{l a}\left(f_{a l}(\infty)-f_{a l}(-\infty)\right) .
$$

For gauge transformations which go to the identity transformation at the spacetime infinity, $f_{a l}(t)$ approaches $2 \pi \times$ (integer) as $t \rightarrow \pm \infty$ and $f_{a l}(t) \rightarrow 0$ as $l \rightarrow \infty$. Thus the above topological charge should be integer even in the noncommutative $U(1)$ gauge theory.

The path integral amplitude for a given configuration is proportional to $\mathcal{A}=e^{i S_{C S}}$, which can change nontrivially under the gauge transformations which go to the identity at spacetime infinity. That change should be unity for the theory to be consistent,

$$
e^{i \Delta S_{C S}}=e^{-i 4 \pi^{2} \kappa \mathcal{N}}=1,
$$

for arbitrary integer $\mathcal{N}$. This consistency leads to the quantization rule

$$
\kappa=\frac{n}{2 \pi}
$$

with an integer $n$. Thus the Chern-Simons coefficient should be quantized for the noncommutative $U(N)$ gauge theory. The parameter $n$ is also called the 'level' of the Chern-Simons term. This quantization rule is identical to that for the commutative theory with larger gauge group but is novel for the noncommutative $U(1)$ theory.

It seems that the gauge transformations considered above do not have the smooth limit when $\theta \rightarrow 0$. One can write the noncommutative version of more familiar map from $R^{3} \rightarrow S U(2)$ with nonzero wrapping. Define a generator of $U(N)$ group

$$
\Theta(z, \bar{z})=\bar{W}(\bar{z}) \frac{1}{W \bar{W}} W(z)
$$

with holomorphic $N$ dimensional row vector $W(z)$ whose components are polynomials of order $l$. Consider a gauge transformation $g=e^{-i f(t) \Theta(\infty)} e^{i f(t) \Theta(z, \bar{z})}$ with the boundary condition that $f( \pm \infty)=2 \pi m_{ \pm}$with integers $m_{ \pm}$. This gauge transformation goes to the identity at spacetime boundary. This gauge transformation has the topological charge $\mathcal{N}=-\left(m_{+}-m_{-}\right) l$ and also a smooth $\theta=0$ limit. The expression (13) has a smooth $\theta=0$ limit except for the $U(1)$ theory. (When $W$ is antiholomorphic, one gets opposite topological number if $W \bar{W}$ is invertible.) A similar expression for the above gauge transformation appears also in the calculation of the topological number for noncommutative $C P(N)$ theories 11 .

A different form of the Chern-Simons action can be obtained by using the covariant position operator

$$
X^{i}=x^{i}+\theta \epsilon_{i j} A_{j} .
$$

These operators appear in the matrix interpretation of the noncommutative Yang-Mills term. The ChernSimons term becomes

$$
S_{C S}^{\prime}=\int d t 2 \pi \theta \operatorname{Tr}\left\{\frac{\kappa}{2 \theta^{2}} \operatorname{tr} \epsilon_{i j} \nabla_{0} X^{i} X^{j}-\frac{\kappa}{\theta} \operatorname{tr} A_{0}\right\},
$$

where $\nabla_{0} X^{i}=\partial_{0} X^{i}-i\left[A_{0}, X^{i}\right]$. This expression is possible only for the noncommutative gauge theory. The first term of the right side is manifestly gauge invariant. The second term is not gauge invariant. Under the local gauge transformation $e^{i \Lambda_{D}}$, it changes identically as before, leading to the same quantization condition. The quantization of the coefficient of $A_{0}$ has appeared before. In the gauged dynamics of quantum mechanics with zero spatial dimension, the term linear in $A_{0}$ is a one-dimensional analogue of the three dimensional Chern-Simons term and the quantization of its coefficient in the $U(1)$ theory has been explored before [12]. The reasons for the quantization in both cases are identical.

This gauge noninvariant term has been studied also in different context. The term proportional to $A_{0}$ is the background electric charge density. As explored in the Maxwell and Chern-Simons theories before [14], one can add an action which describes the effect of uniform background electric charge density. In the Chern-Simons theory, it can be translated to a uniform background magnetic field. The action is

$$
S_{b a c k}=\int d t 2 \pi \theta \operatorname{Tr}\left\{-\rho_{e} \operatorname{tr} A_{0}\right\},
$$

where $\rho_{e}$ is constant. This action is not gauge invariant, and looks identical to the last term for the alternative Chern-Simons action in the previous paragraph. Thus the gauge invariance implies the modification of the quantization rule (12) to

$$
\kappa+\rho_{e} \theta=\frac{n}{2 \pi} .
$$


Only a linear combination of these two parameters is quantized.

The Chern-Simons theories can be used to describe anyons, or particles of fractional spin and statistics. The commutation relation (1) which defines the noncommutativity does not violate the translation and rotation symmetries. Thus, we expect that there exist corresponding conserved quantities. (See Ref. [4]7] for the previous discussion for these conserved quantities.) Under the rotation $x^{i} \rightarrow x^{i}=x^{i}+\delta x^{i}$ with $\delta x^{i}=\epsilon_{i j} x^{j}$, the fields in the commutative theories transform as $\delta \phi(x)=-\delta x^{i} \partial_{i} \phi$ and $\delta A_{i}(x)=\epsilon_{i j} A_{j}-\delta x^{j} \partial_{j} A_{i}$. Here we have dropped the infinitesimal parameter in the front of the variation. We can decompose the transformation of the fields into the gauge covariant one plus a pure gauge transformation 13. In the noncommutative field theories, one should be careful about the ordering of coordinates. Especially, with $\phi^{\prime}\left(x^{\prime}\right)=\phi(x)$ under the rotation, we notice that in the fourier expansion

$$
\begin{aligned}
\delta \phi & =\int d^{2} p \phi(p)\left(e^{i\left(x^{i}-\delta x^{i}\right) p_{i}}-e^{i x^{i} p_{i}}\right) \\
& =-\frac{1}{2}\left(\delta x^{i} \partial_{i} \phi+\partial_{i} \phi \delta x^{i}\right)
\end{aligned}
$$

Here we have used the identity $e^{i\left(x^{i}-\delta x^{i}\right) p_{i}}=$ $e^{-i \delta x^{i} p_{i} / 2} e^{i x^{i} p_{i}} e^{-i \delta x^{i} p_{i} / 2}$ when $\delta x^{i}$ is linear in $x^{i}$. Thus, the generalization of the transformation to the noncommutative case is that the transformation $\delta \phi$ and $\delta A_{i}$ is the sum of the covariant transformation

$$
\begin{aligned}
& \delta_{\text {cov }} \phi=\frac{1}{2} \epsilon_{i j}\left(X^{i} \nabla_{j} \phi+\nabla_{j} \phi x^{i}\right), \\
& \delta_{\text {cov }} A_{i}=-\frac{1}{2}\left(X^{i} F_{12}+F_{12} X^{i}\right),
\end{aligned}
$$

plus a local gauge transformation $\delta_{\text {gauge }} \phi=i \Lambda \phi$ and $\delta_{\text {gauge }} A_{i}=\nabla_{i} \Lambda$ with

$$
\Lambda=\frac{1}{2}\left[\epsilon_{i j}\left(x^{i} A_{j}+A_{j} x^{i}\right)+\theta A_{i}^{2}\right] .
$$

The above expression has the well defined commutative limit as $\theta \rightarrow 0$.

The Noether charge we are interested in is that for the covariant transformation $\delta_{\text {cov }}=\delta-\delta_{\text {gauge }}$. While the action is invariant under $\delta$, the action under $\delta_{\text {gauge }}$ changes by a total time derivative term after the Gauss law is used. This is due to the Chern-Simons term and the background charge. The gauge invariant angular momentum is then

$$
\begin{aligned}
J= & -2 \pi \theta \operatorname{Tr} \operatorname{tr}\left\{\frac{1}{e^{2}} F_{0 i} \delta_{c o v} A_{i}+\nabla_{0} \phi \delta_{c o v} \bar{\phi}+\delta_{c o v} \phi \nabla_{0} \bar{\phi}\right. \\
& \left.+\frac{\rho_{e}}{2}\left(X^{i}\right)^{2} F_{12}\right\} .
\end{aligned}
$$

We have discarded gauge noninvariant boundary terms, which are related to the manifestly conserved current.
This is the generalization of the angular momentum 14] to the noncommutative space. In the symmetric phase $F_{12}$ can have a uniform nonzero value in the symmetric phase, in which case this ground value should be substracted from the above expression. (See for detail in Ref. [14.)

To calculate the angular momentum of the various objects in the theory, let us focus on the $U(1)$ gauge theory without the Maxwell term nor the background charge. The angular momentum then has only the matter contribution. We are interested in finding the explicit angular momentum for an initial configuration of the gauge field and the Higgs field. We assume that the initial velocity or time derivative is zero, but there is nonzero $A_{0}$ to satisfy the Gauss law constraint. In addition we assume that the initial configuration is rotationally symmetric. The most general ansatz with the covariant position operators instead of the gauge field is then

$$
\begin{aligned}
& \phi(x)=\sum_{l=0}^{\infty} f_{l}|l\rangle\left\langle l+\mu\left|, \quad A_{0}(x)=\sum_{l=0}^{\infty} a_{l}\right| l\right\rangle\langle l|, \\
& Z=X+i Y=\sum_{l=0}^{\infty} \sqrt{2 \theta\left(l+1+k_{l}\right)}|l\rangle\langle l+1|,
\end{aligned}
$$

with real coefficients $f_{l}, a_{l}, k_{l}$. We call $\mu$ the vorticity of the Higgs field at origin. This can be positive or negative integer. In the negative case, the coefficients $f_{0}, . ., f_{|\mu|-1}$ vanish. When $A_{i}=0$, the coefficients $k_{l}$ vanish. For localized configurations the coefficient $k_{l}$ should converge to a finite value in the large $l$ limit. Also we choose $k_{-1}=0$. As $F_{12}=-\frac{1}{\theta}+\frac{[Z, \bar{Z}]}{2 \theta^{2}}$, the total magnetic flux $\Psi=2 \pi \theta \operatorname{Tr} F_{12}$ becomes

$$
\Psi=2 \pi \lim _{l \rightarrow \infty} k_{l} \equiv 2 \pi(\mu+\alpha) .
$$

This limit can be positive or negative. Its analogue in the commutative theory is the expression for the magnetic flux as the line integration of the gauge field at the spatial infinity, $\Psi=\oint_{\infty} d l^{i} A_{i}$. The Gauss law $\kappa F_{12}-\rho=0$ where the charge density is $\rho=-i\left(\nabla_{0} \phi \bar{\phi}-\phi \nabla_{0} \bar{\phi}\right)$ becomes

$$
\frac{\kappa}{\theta}\left(k_{l}-k_{l-1}\right)=-2 f_{l}^{2} a_{l} \text {. }
$$

for all $l$. The conserved electric charge $Q=2 \pi \theta \operatorname{Tr} \rho=\kappa \Psi$ is

$$
Q=2 \pi \kappa(\mu+\alpha)
$$

In the symmetric phase, the electric charge is quantized in integer in quantum mechanics as the field is invariant under $2 \pi$ phase rotation. A configuration without vorticity describes a collection of elementary particles. A single particle would have the values $\mu=0$ and $\alpha=1 /(2 \pi \kappa)=1 / n$, and so its magnetic flux is fractional.

The angular momentum in the theory without the Maxwell term can be rewritten as 


$$
\begin{aligned}
& J=- i \pi \operatorname{Tr}\left\{\left(X^{i}\right)^{2}\left(\nabla_{0} \phi \bar{\phi}-\phi \nabla_{0} \bar{\phi}\right)\right. \\
&\left.-\left(\nabla_{0} \phi\left(x^{i}\right)^{2} \bar{\phi}-\phi\left(x^{i}\right)^{2} \nabla_{0} \bar{\phi}\right)\right\} .
\end{aligned}
$$

The angular momentum for the rotationally symmetric ansatz becomes

$$
J=\pi \kappa\left(\alpha^{2}-\mu^{2}\right) .
$$

This expression is identical to one appeared in Ref. [15]. When this result is combined with the quantization rule, the consequence is fascinating.

In the symmetric phase, our initial configuration is a generic one which may describe a q-ball with vortices in the middle. Many elementary particles could make qballs. Elementary particle has charge quantized. In noncommutative theory, the charged matter field can carry only unit charge. Thus, the magnetic flux is fractional $1 / \kappa=2 \pi / n$. The spin of elementary particles and antiparticles with $\alpha= \pm 1 / 2 \pi \kappa$ and $\mu=0$ is identical to

$$
s_{\text {particle }}=\frac{1}{2 n},
$$

which has a fractional spin as $n$ is an integer. In the commutative case, their statistics comes from the AharonovBohm phase. We expect that is true even in the noncommutative case.

In the broken phase, the scalar field has nonzero expectation value, $\lim _{l \rightarrow \infty} f_{l}=v$. For finite energy configurations, the gradient energy $\nabla_{i} \phi \nabla_{i} \bar{\phi}$ should vanish quickly, which implies that $k_{l} \rightarrow \mu$ or $\alpha=0$. Then the magnetic flux is determined by the vorticity $\Psi=2 \pi \mu$ as in the commutative case. Elementary vortices and antivortices with $\alpha=0, \mu=1$ carry the spin

$$
s_{\text {vortex }}=-\frac{n}{2} \text {. }
$$

Thus vortices in the broken phase can be fermions, bosons. The sign difference between the spin of elementary particles and that of vortices appears also in the commutative case. The statistics of vortices is expected to be due to the Aharonov-Bohm phase plus the Magnus phase, as in the commutative case [15.

We have shown that the Chern-Simons coefficient is quantized on noncommutative space. When the background charge is introduced, only a linear combination of the Chern-Simons coefficient is quantized. Furthermore, we argued that elementary particles in the symmetric phase can have fractional spin but vortices in the broken phase can be only fermions or bosons.

\section{Acknowledgment}

This work is supported in part by KOSEF 1998 Interdisciplinary Research Grant 98-07-02-07-01-5 (DB and KL) and by UOS Academic Research Grant (DB). Note Added: Up on finishing this paper, a related paper [16] appeared in the hep-th.
[1] A. Connes, M.R. Douglas and A. Schwartz, Noncommutative geometry and matrix compactification on tori, JHEP 9802 (1992) 003, hep-th/9711162, N. Seiberg and E. Witten, String theory and noncommutative geometry, JHEP 09 (1999) 032, hep-th/9908142.

[2] N. Ishibashi, S. Iso, H. Kawai and Y. Kitazawa, Nucl. Phys. B 573 (2000) 573, hep-th/9910004; J. Ambjorn, Y.M. Makeenkom J. Nishimura and R.J. Szabo, Lattice gauge fields and discrete noncommutative Yang-Mills theory, JHEP 0005 (2000) 023, hep-th/0004147; D.J. Gross, A. Hashimoto and N. Itzhaki, Observables of noncommutative gauge theories, hep-th/0008075.

[3] J. Madore, S. Schraml, P. Schupp and J. Wess, Eur. Phys. J. C16 (2000) 161, hep-th/0001203.

[4] D. Bak, K. Lee and J.-H. Park, Comments on noncommutative gauge theories., hep-th/0011244.

[5] C-S. Chu. Induced Chern-Simons and WZW action in noncommutative spacetime, Nucl. Phys. B580 (2000) 352, hep-th/0003007, A.A. Bichl, J.M. Grimpstrup, V. Putz, and M Scheda, Perturbative Chern-Simons Theory on noncommutative $R^{3}$, hep-th/0004071; G.-H. Chen and Y.-S. Wu, One loop shift in noncommutative Chern-Simons coupling, hep-th/0006114; A.P. Polychonakos, Noncommutative Chern-Simons terms and the noncommutative vacuum, hep-th/0010264; N. Grandi and G.A. Silva, Chern-Simons action in noncommutative space, hep-th/0010113; J. Kluson, Matrix model and noncommutative Chern-Simons theory, hep-th/0012184.

[6] G.S. Lozano, E.F. Moreno and F.A. Schaposnik, Selfdual Chern-Simons Solitons in noncommutative space, hep-th/0012266; A. Khare and M.B. Paranjape, Solitons in $2+1$ dimensional noncommutative Maxwell-ChernSimons Higgs theories, hep-th/0102016.

[7] D. Bak, S.K. Kim, K.-S. Soh and J.H. Yee, Noncommutative Chern-Simons Solitons, hep-th/0102137.

[8] M.M. Sheikh-Jabbari, A note on noncommutative ChernSimons theories, hep-th/0102092.

[9] L. Susskind, The quantum Hall fluid and noncommutative Chern-Simons theories, hep-th/0101029.

[10] S. Deser, R. Jackiw and S. Templeton, Three dimensional massive gauge theories, Phys. Rev. Lett. 48 (1983) 975; Topologically massive gauge theories, Ann. Phys. NY 140 (182) 372.

[11] B-H. Lee, K. Lee and H.S. Yang, The $C P(n)$ model on noncommutative plane Phys. Lett. B498 (2001) 277, hepth/0007140.

[12] G. Dunne, K. Lee and C. Lu, On the finite temperature Chern-Simons coefficient, Phys. Rev. Lett. 78 (1997) 3434.

[13] R. Jackiw, Phys. Rev. Lett. 41 (1978) 1635.

[14] K. Lee, Phys. Rev. D49 (1994) 4265; K. Lee and P. Yi, Phys. Rev. D52 (1995) 2412.

[15] Y. Kim and K. Lee, Phys. Rev. 49 (1994) 2041.

[16] V.P. Nair and A.P. Polychronakos, On level quantization for the noncommutative Chern-Simons theory, hepth/0102181. 